Journal of Universal Mathematics

Vol.3 No.2 PP.131-136 (2020)

ISSN-2618-5660

DOI: $10.33773 /$ jum.637104

\title{
REMARKS ON THE ARITHMETICAL FUNCTION $\left(a_{p}(n)\right)$
}

\author{
A. AWEL
}

\begin{abstract}
In this paper, for any arbitrary two primes $p$ and $q$ the relationship between the corresponding arithmetic functions $\left(a_{p}(n)\right)$ and $\left(a_{q}(n)\right)$ are investigated. Furthermore, a general formula for statistical density of all sets on which the two arithmetic function have the same value is also established.
\end{abstract}

\section{INTRODUCTION}

In [3] and [6], Fast and Steinhaus introduced the concept of statistical convergence, independently. In [10], Zygmund gave a name "almost convergence" to the this concept and established a relation between statistical convergence and strong summability of sequences. Especially in [7], Schoenberg gave a matrix characterization of the statistical convergence.

Let $K$ be a subset of the positive integers $\mathbb{N}$ and $K_{n}:=\{k \leq n: k \in K\}$. Natural density of the set $K$ is given by

$$
\delta(K):=\lim _{n \rightarrow \infty} \frac{\left|K_{n}\right|}{n}
$$

provided that this limit exists. The symbol $|A|$ denotes the cardinality of the set $A$.

A real number sequence $x=\left(x_{k}\right)_{k=1}^{\infty}$ is statistically convergent to $L$ provided that for every $\varepsilon>0$ the set

$$
K(\varepsilon)=\left\{k \in \mathbb{N}:\left|x_{k}-L\right| \geq \varepsilon\right\}
$$

has a natural density of zero. In this case, we write $s t-\lim x=L$.

An arithmetic function is any real or complex valued function defined on a set of positive integers. In analytic number theory arithmetic functions are simple but very useful tools to understand many advance concepts. There are a lot of arithmetical function but here, we are interested in only the arithmetic function $\left(a_{p}(n)\right)$. The main tool here is the concept of the natural (or asymptotic) density. Some more related results about arithmetical functions can be found in [2], [4] and [5], etc.

Date: October, 2019.

2000 Mathematics Subject Classification. 40A35, 11B05, 11425.

Key words and phrases. Statistical limit and cluster points, Natural density, Arithmetical function. 
The elementary properties of the arithmetic function $\left(a_{p}(n)\right)$ is studied in [8] by T. Salat in the sense of natural density. Later on, the same arithmetical function with the perspective of ideal convergence has been investigated in [4].

Very recently the statistical limit and cluster points of the sequence $\left(a_{p}(n)\right)$ and some others were studied in [1].

In this paper, by taking different primes $p$ and $q$ the relationship between the arithmetical functions $\left(a_{p}(n)\right)$ and $\left(a_{q}(n)\right)$ will be studied and a formula will be produced for the natural density of the sets having same value on both functions is established.

Definition 1. Let $p$ be a prime number. The arithmetic function $a_{p}(n)$ is defined as follows: $a_{p}(1)=0$ and if $n \geq 1$, then $a_{p}(n)$ is the unique positive integer $j \geq 0$ satisfying $p^{j} \mid n$ but not $p^{j+1} \mid n$.

For example for $p=3$ the sequence $\left(a_{p}(n)\right)$ is given as follows:

$$
\left(a_{3}(n)\right)=(0,0,1,0,0,1,0,0,2,0,0,1,0,0,1,0,0,1,0,0,1, \ldots) .
$$

In the proof of the main results, we need following Lemmas:

Lemma 1.1. [9] If $A$ and $B$ are two mutually disjoint subsets of the set natural numbers $\mathbb{N}$, then

$$
\delta(A \cup B)=\delta(A)+\delta(B)
$$

holds.

Lemma 1.2. [9] Let $S=\left\{s_{1}, s_{2}, \ldots, s_{n}, ..\right\}$ be a subset of the set of natural numbers $\mathbb{N}$ where $s_{1}<s_{2}<\ldots<s_{n}<\ldots$ satisfied. Then,

$$
\delta(S)=\lim _{n \rightarrow \infty} \frac{\left|S\left(s_{n}\right)\right|}{s_{n}} .
$$

\section{Main Results}

In this section, we will see the key results of the paper for any two primes $p$ and $q$. First of all, let us defined the following sets which are associated with $p$ and $q$ :

$$
\begin{gathered}
K_{0}:=\{n \in \mathbb{N}:(n, p)=1 \text { and }(n, q)=1\}, \\
K_{1}:=\left\{n \in \mathbb{N}: n=p k_{i} \text { or } n=q k_{i} \text { where } k_{i} \in K_{0}\right\}, \\
K_{2}:=\left\{n \in \mathbb{N}: n=p^{2} k_{i} \text { or } n=q^{2} k_{i} \text { where } k_{i} \in K_{0}\right\}, \\
K_{3}:=\left\{n \in \mathbb{N}: n=p^{3} k_{i} \text { or } n=q^{3} k_{i} \text { where } k_{i} \in K_{0}\right\}, \\
\vdots \\
K_{j}:=\left\{n \in \mathbb{N}: n=p^{j} k_{i} \text { or }, n=q^{j} k_{i} \text { where } k_{i} \in K_{0}\right\}
\end{gathered}
$$

and so on.

Also, let us denote the union of these sets by

$$
D:=\bigcup_{j=1}^{\infty} K_{j} .
$$

Lemma 2.1. For each $i \neq j$, the sets $K_{i}$ and $K_{j}$ defined above are mutually disjoint subset of natural numbers. 
Proof. Let $n \in K_{i}$ be an arbitrary element, then either $n=p^{i} k_{1}$ or $n=q^{i} k_{1}$ where $k_{1} \in K_{0}$. Let us see each case one by one.

Case 1: Assume that $n=p^{i} k_{1}$. For $j \neq i$ suppose that $n \in K_{j}$, then we have also two cases: either $n=p^{j} k_{2}$ or $n=q^{j} k_{2}$ where $k_{2} \in K_{0}$. Suppose $n=p^{j} k_{2}$.

If $i<j$, then $n=p^{j} k_{2}=p^{i} p^{j-i} k_{2}=p^{i} k_{1}$ this implies that $k_{1}=p^{j-i} k_{2}$ and this also in turn implies that $k_{1}$ could not be in $K_{0}$ which contradicts to our assumption $n \in K_{0}$. Hence, $n \notin K_{j}$.

If we also consider $i>j$, then $n=p^{i} k_{1}=p^{j} p^{i-j} k_{1}=p^{j} k_{2}$. This implies that $k_{2}=p^{i-j} k_{1}$ and hence $k_{2}$ could not be in $K_{0}$ which contradicts to our assumption $k_{2} \in K_{0}$.

If $n=q^{j} k_{2}$ with $i<j$, then $n=q^{j} k_{2}=q^{i} q^{j-i} k_{2}=q^{i} k_{1}$ and this implies that $k_{1}=q^{j-i} k_{2}$. It simply means that $k_{1}$ could not be in $K_{0}$ which contradicts to our assumption $k_{1} \in K_{0}$. Hence, $n \neq q^{j} k_{2}$.

If we also consider $i>j$, then $n=p^{i} k_{1}=p^{j} p^{i-j} k_{1}=p^{j} k_{2}$. This implies that $k_{2}=p^{i-j} k_{1}$ and hence $k_{2}$ could not be in $K_{0}$ which contradicts to our assumption $k_{2} \in K_{0}$. Therefore, $n \notin K_{j}$.

Case 2: Assume that $n=q^{i} k_{1}$.

For $j \neq i$ suppose that $n \in K_{j}$, then we have also two cases either $n=p^{j} k_{2}$ or $n=q^{j} k_{2}$ where $k_{2} \in K_{0}$. Suppose $n=p^{j} k_{2}$ holds. If $i<j$, then $n=p^{j} k_{2}=$ $p^{i} p^{j-i} k_{2}=p^{i} k_{1}$ this implies that $k_{1}=p^{j-i} k_{2}$. This also in turn implies that $k_{1}$ could not be in $K_{0}$ which contradicts to our assumption $k_{1} \in K_{0}$. Hence, $n \neq p^{j} k_{2}$.

If we also consider $i>j$, then $n=p^{i} k_{1}=p^{j} p^{i-j} k_{1}=p^{j} k_{2}$. This implies that $k_{2}=p^{i-j} k_{1}$ and hence $k_{2}$ could not be in $K_{0}$ which contradicts to our assumption $k_{2} \in K_{0}$. Therefore, on each case $n \neq p^{i} k_{1}$.

If $n=q^{j} k_{2}$ with $i<j$, then $n=q^{j} k_{2}=q^{i} q^{j-i} k_{2}=q^{i} k_{1}$. This implies that $k_{1}=q^{j-i} k_{2}$ this simply means that $k_{1}$ could not be in $K_{0}$ which contradicts to our assumption $n \in K_{0}$. Hence, $n \neq q^{j} k_{2}$.

If we consider $i>j$, then $n=p^{i} k_{1}=p^{j} p^{i-j} k_{1}=p^{j} k_{2}$. This implies that $k_{2}=p^{i-j} k_{1}$ and hence $k_{2}$ could not be in $K_{0}$ which contradicts to our assumption $k_{2} \in K_{0}$. For $k_{1} \in K_{0}, n \neq p^{i} k_{1}$. Therefore, in all case $n \notin K_{j}$. Hence, the sets are disjoint and this completes the proof.

Lemma 2.2. The set $D$ defined in (2.1) is exactly the same as the set

$$
C:=\{n: n=p k \text { or } n=q k, k \in \mathbb{N}\} .
$$

That is, we have $D=C$.

Proof. Let $n \in D$. By Lemma 2.1 the sets $K_{i}$ 's are mutually disjoint. Then, for a fixed $i \in \mathbb{N}$ we have $n \in K_{i}$. Definition of $K_{i}$ 's, implies two cases $n=p^{i} k_{0}$ or $n=q^{i} k_{0}$ where $k_{0} \in K_{0}$.

Case 1: $n=p^{i} k_{0}$. Now, if $i=1$ then obviously $n \in C$. If $i>1$ then $n=p\left(p^{i-1} k_{0}\right)$. This implies that for $k_{2}=p^{i-1} k_{0}$. So, we have $n=p k_{2}$ which also implies $n \in C$. Hence, $D \subseteq C$.

Case 2: $n=q^{i} k_{0}$. Now, if $i=1$ then obviously $n \in C$. If $i>1$, then $n=q\left(q^{i-1} k_{0}\right)$. This implies that for $k_{2}=q^{i-1} k_{0}$. So, we have $n=q k_{2}$ which also implies $n \in C$. Hence, in each case we have $D \subseteq C$.

To prove the converse side, let $n \in C$ then, $n=p k$ where $k \in \mathbb{N}$. Now, if $(p, k)=1$, then we have $n \in K_{1}$. If $(p, k) \neq 1$, then $(p, k)=p$ this implies $n=p^{2} k_{2}$. If $\left(p, k_{2}\right)=1$ then, $n \in K_{2}$. Since $n$ a is fixed number, then if we continue in this 
way we will stop at some point $i \in \mathbb{N}$ such that $n=p^{i} k_{i}$ and $\left(k_{i}, p\right)=1$ which implies that $n \in K_{i}$. Therefore, we have also $C \subseteq D$. This completes the proof.

Theorem 2.3. For the set $K_{0}:=\left\{n \in \mathbb{N}: a_{p}(n)=1=a_{q}(n)\right\}$, we have

$$
\delta\left(K_{0}\right)=\frac{(p-1)(q-1)}{p q} .
$$

Proof. By Lemma 2.2 we have,

$$
D=\bigcup_{i=1}^{\infty} K_{i}=A \cup B
$$

where

$$
A=\{n: n=p k, k \in \mathbb{N}\} \text { and } B=\{n: n=q k, k \in \mathbb{N}\} .
$$

From the definitions of the sets, we have $\mathbb{N} \backslash\{1\}=K_{0} \cup D$ and $K_{0} \cap D=\emptyset$ hold. Also,

$$
1=\delta(\mathbb{N})=\delta(D)+\delta\left(K_{0}\right)
$$

and

$$
\delta(D)=\delta(A \cup B)=\delta(A)+\delta(B)-\delta(A \cap B)=\frac{1}{p}+\frac{1}{q}-\frac{1}{p q}
$$

clearly satisfied. Therefore, we have

$$
\delta\left(K_{0}\right)=1-\left(\frac{1}{p}+\frac{1}{q}-\frac{1}{p q}\right)=\frac{p q+1-(p+q)}{p q}=\frac{(p-1)(q-1)}{p q}
$$

holds. Hence, this completes the proof.

Theorem 2.4. For each $j \in \mathbb{N}$, the natural density of $K_{j}$ defined above is given by the following formula:

$$
\delta\left(K_{j}\right)=\left(\frac{1}{p^{j}}+\frac{1}{q^{j}}\right) \frac{(p-1)(q-1)}{p q}
$$

Proof. For the sake of convenience let us denote

$$
K_{j}:=A_{j} \cup B_{j}
$$

where

$$
A_{j}=\left\{n \in \mathbb{N}: n=p^{j} k_{i}, k_{i} \in K_{0}\right\} \text { and } B_{j}=\left\{m \in \mathbb{N}: m=q^{j} k_{i}, k_{i} \in K_{0}\right\} .
$$

It is clear from the definition of the sets for each $p \neq q$,

$$
A_{j} \cap B_{j}=\emptyset \text {. }
$$

Let $S_{n}:=\left\{k_{1}^{0}, k_{2}^{0}, k_{3}^{0}, \ldots, k_{n}^{0}\right\}$ be the set of first $n$ elements of $K_{0}$ satisfying $k_{1} \leq k_{2} \leq k_{3} \leq \cdots \leq k_{n}$.

As a result of Theorem 2.3 and definition of density, we have

$$
\delta\left(K_{0}\right)=\lim _{n \rightarrow \infty} \frac{\left|K_{0} \cap S_{n}\right|}{k_{n}^{0}}=\frac{(p-1)(q-1)}{p q}
$$

and clearly $\delta\left(K_{j}\right)=\delta\left(A_{j}\right)+\delta\left(B_{j}\right)$. For the set

$$
A_{j}:=\left\{n \in \mathbb{N}: n=p^{j} k, k \in K_{0}\right\}
$$


$j \in \mathbb{N}$, we have

$$
\left|A_{j} \cap\left(p^{j} S_{n}\right)\right|=\left|K_{0} \cap S_{n}\right|=n .
$$

Hence,

$$
\begin{gathered}
\delta\left(A_{j}\right)=\lim _{n \rightarrow \infty} \frac{\left|A_{j} \cap\left(p^{j} S_{n}\right)\right|}{p^{j} k_{n}^{0}}=\frac{1}{p^{j}} \lim _{n \rightarrow \infty} \frac{\left|K_{0} \cap S_{n}\right|}{k_{n}^{0}}= \\
=\frac{1}{p^{j}} \delta\left(K_{0}\right)=\frac{1}{p^{j}} \frac{(p-1)(q-1)}{p q}
\end{gathered}
$$

Similarly for the set $B_{j}:=\left\{n \in \mathbb{N}: n=q^{j} k\right.$ where $j \in \mathbb{N}$ and $\left.k \in K_{0}\right\}$. We have

$$
\left|B_{j} \cap\left(q^{j} S_{n}\right)\right|=\left|K_{0} \cap S_{n}\right|=n,
$$

then

$$
\begin{aligned}
\delta\left(B_{j}\right)= & \lim _{n \rightarrow \infty} \frac{\left|B_{j} \cap\left(q^{j} S_{n}\right)\right|}{q^{j} k_{n}^{0}}=\frac{1}{q^{j}} \lim _{n \rightarrow \infty} \frac{\left|K_{0} \cap S_{n}\right|}{k_{n}^{0}} \\
& =\frac{1}{q^{j}} \delta\left(K_{0}\right)=\frac{1}{q^{j}} \frac{(p-1)(q-1)}{p q}
\end{aligned}
$$

satisfied. Since $\delta\left(K_{i}\right)=\delta\left(A_{i} \cup B_{i}\right)=\delta\left(A_{i}\right) \cup \delta\left(B_{i}\right)$ the result follows immediately.

\section{Application}

In this section, for a specific values of $p$ and $q$, we will denote the relationship between $\left(a_{p}(n)\right)$ and $\left(a_{q}(n)\right)$ by applying the above main results.

Now, let us consider the primes $p=3$ and $q=5$ and the arithmetic functions associated with them:

$$
\begin{aligned}
& \left(a_{3}(n)\right)=(0,0,1,0,0,1,0,0,2,0,0,1,0,0,1,0,0,1,0,0,1,0,0,1, \ldots) \\
& \left(a_{5}(n)\right)=(0,0,0,0,1,0,0,0,0,1,0,0,0,0,1,0,0,0,0,1,0,0,0,0, \ldots)
\end{aligned}
$$

Therefore, associated sets

$$
\begin{gathered}
K_{0}:=\{n \in \mathbb{N}:(n, 3)=1 \text { and }(n, 5)=1\}, \\
K_{1}:=\left\{n \in \mathbb{N}: n=3 k_{i} \text { or } n=5 k_{i} \text { where } k_{i} \in K_{0}\right\}, \\
K_{2}:=\left\{n \in \mathbb{N}: n=3^{2} k_{i} \text { or } n=5^{2} k_{i} \text { where } k_{i} \in K_{0}\right\}, \\
K_{3}:=\left\{n \in \mathbb{N}: n=3^{3} k_{i} \text { or } n=5^{3} k_{i} \text { or } k_{i} \in K_{0}\right\}, \\
\vdots \\
K_{j}:=\left\{n \in \mathbb{N}: n=3^{j} k_{i} \text { or } n=5^{j} k_{i} \text { or } k_{i} \in K_{0}\right\} .
\end{gathered}
$$

Then, by the results of Theorem 2.3 and Theorem 2.4 we have

$$
\delta\left(K_{0}\right)=\frac{(5-1)(3-1)}{15}=\frac{8}{15}
$$

and for each $j \in \mathbb{N}$

hold, respectively.

$$
\delta\left(K_{j}\right)=\left(\frac{1}{3^{j}}+\frac{1}{5^{j}}\right) \frac{8}{15}
$$




\section{Conclusion and Recommendation}

For any two distinct prime $p$ and $q$, we have established a formula for the asymptotic density of the set of points on which the corresponding arithmetic functions $\left(a_{p}(n)\right)$ and $\left(a_{q}(n)\right)$ have the same value (for any arbitrary number) $m \in \mathbb{N}$

This paper has established a relationship between the arithmetic functions $a_{p}(n)$ and $a_{q}(n)$ for two different prime numbers in terms of natural density. Let $p_{1}, p_{2}, \ldots, p_{m}$ distinct prime numbers and consider the sets

$$
K_{0}:=\left\{n \in \mathbb{N}:\left(n, p_{i}\right)=1 \text { for all } i=1,2, \ldots, m\right\}
$$

and for any $j \in \mathbb{N}$

$$
K_{j}:=\left\{n \in \mathbb{N}: n=p_{i}^{j} k_{l} \text { for any } k_{l} \in K_{0} \text { and for all } i=1,2, \ldots, m\right\} .
$$

By using similar ways an interested author could extend the result of this paper to find

and

$$
\delta\left(K_{0}\right)=?
$$

for all $j \in \mathbb{N}$.

$$
\delta\left(K_{j}\right)=?
$$

\section{ACKNowledgment}

I would like to thank Prof. Dr. M. Küçükaslan for his guidance and valuable contributions during the creation and solution of the problem.

I would also like to thank the referees who contributed to the study with their precious warnings.

\section{REFERENCES}

[1] Abdu Awel and M. Küçükaslan, (2020) A note on statistical limit and cluster points of the arithmetical functions $a_{p}(n), \gamma(n), \tau(n)$, J. Indones. Math. Soc. (accepted for publication).

[2] V. Bal'az, J. Gogola, T. Visnyai, (2018) $I_{c}^{q}$-convergence of arithmetical functions. J. Number Theory, vol. 183, 74-83

[3] Fast, H., (1951) Sur la convergence statistique., Colloq.Math, vol. 2, 241-244.

[4] Fehéra, Z., Lászlóa, B., Mačajb, M., Šalát, T., (2006) Remarks on arithmetical functions $a_{p}(n), \gamma(n), \tau(n)$, Annales Mathematicae et Informaticae, vol 33, 35-43.

[5] Janos T. T., Ferdinand F., Jozsef B., Laszlo Z., (2020), On $I_{<_{q}}$ and $I_{\leq_{q}}$ convergence of arithmetic functions, Periodica Mathematica Hungarica, 1-12.

[6] Steinhaus H., (1951) Sur la convergence ordinaire at la convergence asymptotique, Colloc. Math. 2.1, 73-74.

[7] Schoenberg, I. J., (1959) The integrability of certain functions and related summability methods, matrix characterization of statistical convergence, Amer.Math., vol. 66, 361-375.

[8] Šalát, T., (1994) On the function $a_{p}, p^{a_{p}(n)} \backslash \backslash n(n>1)$, Mathematica Slovaca, vol. 44, Number 2, 143-151.

[9] Milan, P., (2017) Density and related topics, Mathematics Institute Slovak Acadamic of Sciences.

[10] Zygmund, A., (1979) Trigonometric series, 2nd., Ed. Vol. II, Cambridge Univ. press, London and New York.

Mekelle University, Mathematics Department, 231, Mekelle, Ethiopia

Email address: abdua90@gmail.com 